\title{
¿Bajo desempeño o malas decisiones? La desigualdad de oportunidades educativas desde una perspectiva comparada
}

\author{
Andrea Cuenca ${ }^{1}$ \\ Investigadora de la Universidad de los Andes, Bogotá, Colombia \\ andreacuencah@gmail.com
}

\section{Jackson, Michelle (Ed.). Determined to Succeed? Performance versus Choice in Educational Attainment. Stanford: Stanford University Press, 2013}

La desigualdad de oportunidades educativas asociada a la clase social de origen ha sido un tema central en el área investigativa sobre estratificación y educación. Un hallazgo frecuente de los estudios en esta área consiste en que, en sociedades industrializadas, los individuos provenientes de familias con nivel socioeconómico alto reciben "más" y "mejor" educación que aquellos con orígenes socioeconómicos más bajos. Como consecuencia, los estudiantes de origen social menos aventajado no solo tienden a presentar un menor rendimiento, sino también a elegir trayectorias educativas menos ambiciosas que sus contrapartes de origen social privilegiado (Breen et al. 2009; Shavit y Blossfeld 1993).

Ese es el tema que aborda el libro Determined to Succeed?, editado por Michelle Jackson. Este libro constituye el primer estudio comparado que analiza simultáneamente el papel de dos factores en la generación de desigualdades socioeconómicas en los logros educativos: el desempeño y las decisiones educativas. El interrogante central que orienta este trabajo es en qué medida la desigualdad de oportunidades educativas puede atribuirse a (1) las diferencias en el

$1 \quad$ Psicóloga. Magíster en Investigación en Educación. 
desempeño académico entre grupos socioeconómicos o (2) a las diferencias en las decisiones educativas que toman los estudiantes pertenecientes a estos grupos, una vez controlado el efecto del desempeño. A lo largo de ocho capítulos, esta pregunta guía los ejercicios empíricos desarrollados por diferentes autores para los casos de Estados Unidos y siete países de Europa occidental.

El capítulo introductorio, escrito por Jackson, discute los principales planteamientos teóricos que subyacen a la división entre desempeño y decisiones educativas. Históricamente, su origen proviene de la distinción entre efectos primarios y secundarios, establecida por el sociólogo francés Raymond Boudon en la década de 1970. Jackson argumenta, sin embargo, que en realidad fueron Girard y Bastide (1963) quienes acuñaron por primera vez los términos, diez años antes. Con todo, la importancia de Boudon radica no solamente en haber elaborado una teoría general sobre la desigualdad de oportunidades educativas, sino que además fue el primero en dar tratamiento sistemático a estos conceptos y hacerlos visibles en su libro Education, Opportunity, and Social Inequality (1974), publicado originalmente en francés bajo el título L'inegalité des Chances en 1973. Si bien los términos efectos primarios y secundarios no son los más apropiados para hacer referencia a los efectos del desempeño y las decisiones, estos han sido derivados de conceptos estadísticos básicos que corresponderían a los efectos indirectos y directos del origen social sobre el logro educativo (Blau y Duncan 1967), respectivamente.

Analizar la desigualdad de oportunidades educativas como resultado de la combinación entre efectos primarios y secundarios tiene dos claras ventajas. Por una parte, permite identificar con detalle los mecanismos subyacentes a la desigualdad. Y por otra, permite discernir las implicaciones concretas de distintas políticas públicas.

En cuanto al primer punto, en la literatura se han identificado diversos mecanismos para dar cuenta de las diferencias en el desempeño. En particular, el libro analiza los mecanismos relacionados con las características económicas y sociales del hogar de origen. Sin embargo, otros mecanismos incluyen: factores genéticos, psicológicos y culturales, condiciones de salud y nutrición, posición entre hermanos y patrones culturales promovidos por las instituciones educativas. Por otra parte, las diferencias en las decisiones educativas pueden comprenderse con base en la interacción de tres mecanismos: los costos de las alternativas educativas en función de los recursos económicos de las familias, los beneficios asociados a dichas alternativas y las propias expectativas de éxito. Estos tres componentes hacen parte de los modelos de elección racional (rational choice) en la toma de decisiones educativas (Breen y Goldthorpe 1997). Al respecto, si bien Jackson afirma que no es necesario acoger la perspectiva teórica de la elección racional para explicar cómo se generan los efectos secundarios, el libro presenta una clara orientación hacia dicha aproximación. Mientras los modelos de elección racional plantean que las decisiones educativas están guiadas por el comportamiento de los individuos para evitar la movilidad hacia abajo, las teorías de la reproducción (Bourdieu y Passeron 1977) enfatizan, como explicativos de estas decisiones, los diferentes valores y normas fomentados dentro de cada grupo social. El componente normativo de las decisiones educativas, sin embargo, no es contemplado en el libro. 
En cuanto al segundo punto, un estudio de esta naturaleza permite identificar las implicaciones de distintas políticas públicas. Las intervenciones enfocadas en los efectos primarios parten del supuesto según el cual para reducir las desigualdades en los logros educativos se requiere reducir las desigualdades en el desempeño académico. Esta alternativa ha sido adoptada en muchos países, donde las intervenciones incluyen ampliar la cobertura en educación preescolar o prestar ayuda económica a las familias con niños de corta edad, como medidas de reducción temprana de las desigualdades en el rendimiento. Por el contrario, centrarse en los efectos secundarios conlleva intervenciones dirigidas a influir las percepciones de los estudiantes y sus familias sobre los costos y beneficios de determinadas transiciones educativas. Jackson argumenta que la segunda opción no solo tendría mayor impacto que la primera, sino que además es más factible en tanto los mecanismos subyacentes a los efectos secundarios son más susceptibles de ser modificados.

El segundo capítulo, escrito por Christiana Kartsonaki, Michelle Jackson y David Cox, trata asuntos metodológicos y propone una operacionalización de los efectos primarios y secundarios. En términos generales, esta consiste en manipular las distribuciones en el desempeño según clase social y en las probabilidades de lograr ciertas transiciones educativas, con el objetivo de obtener una estimación cuantitativa de la contribución de los efectos primarios y secundarios a la desigualdad de oportunidades. Se expone detalladamente el método mediante el cual se descomponen las razones de momios que describen las desigualdades entre grupos socioeconómicos en efectos primarios y secundarios, y se calcula un porcentaje estimado de la importancia relativa de ambos efectos.

En los capítulos tres a diez se presentan los estudios de ocho casos nacionales: Martin Neugebauer, David Reimer, Steffen Schindler y Volker Stocké analizan las transiciones hacia la educación secundaria y terciaria en Alemania; Charlotte Büchner y Rolf van der Velden realizan un estudio acerca del impacto del origen social sobre el logro educativo en el curso del tiempo en Holanda; Mathieu Ichou y Louis-André Vallet evalúan las contribuciones relativas del desempeño y las decisiones a las desigualdades educativas en Francia a lo largo de cuatro décadas; Dalit Contini y Andrea Scagni son los autores del capítulo sobre Italia; Frida Rudolphi realiza un análisis de las transiciones hacia la educación secundaria alta y la terciaria en un rango amplio de cohortes en Suecia; Anders Holm y Mads Meier Joeger trabajan el capítulo sobre Dinamarca; Michelle Jackson es la autora del estudio dedicado a Inglaterra; y por último, Stephen Morgan, Michael Spiller y Jennifer Todd se encargan del estudio sobre Estados Unidos. En cada caso, los autores llevan a cabo un estudio empírico que permita responder a la pregunta central del libro. Empleando los mejores datos disponibles representativos en el ámbito nacional, cada capítulo sigue una estructura uniforme: descripción del contexto institucional, incluyendo la identificación de las transiciones educativas relevantes en el sistema nacional de educación; revisión de investigaciones previas; especificación de los datos, variables y modelos utilizados; resultados y conclusiones.

Las fuentes de datos, así como el tamaño de las muestras y las cohortes de edad difieren según países. Entre las fuentes empleadas se incluyen registros censales, bases de datos transversales o longitudinales (provenientes de estudios 
de cohorte). Las cohortes de edad analizadas oscilan entre las décadas de 1950 y 1990, siendo posible en algunos países realizar un análisis de los cambios en función del tiempo. Pese a las diferencias, los datos en general son suficientes y de calidad para arrojar estimaciones precisas sobre la desigualdad de oportunidades educativas.

Con el objetivo de unificar y facilitar la comparación, los estudios siguen criterios homogéneos en cuanto a selección muestral, construcción de variables y aproximación metodológica. Los autores emplean la clase social (a partir del modelo de Erikson, Goldthorpe y Portocarero EGP) y la escolaridad de los padres como medidas del origen social cuando esta información está disponible; de no ser así, se toma al menos una de estas dos variables. Para los efectos primarios se utiliza una medida de desempeño académico en pruebas estandarizadas o calificaciones escolares cuyo puntaje sea conocido por los estudiantes. En cuanto a los efectos secundarios, se analizan las transiciones educativas. Dado que estas transiciones varían en cada país, se analizan dos transiciones análogas en los casos examinados: el paso de la educación obligatoria al nivel educativo inmediatamente superior (normalmente a la edad de 16 años) y el paso de la educación secundaria alta a la superior (normalmente a los 18 años). Adicionalmente, para los casos de Alemania y Holanda se analiza una transición adicional que tiene lugar en un momento temprano de la trayectoria educativa (a los 10 años de edad).

Los resultados muestran que la magnitud de la desigualdad de oportunidades educativas, así como de los efectos primarios y secundarios, difiere según el país, la transición y el curso del tiempo. Aunque si bien el impacto relativo de los efectos primarios y secundarios varía, la importancia de ambos efectos es transversal.

El último capítulo, escrito por la editora junto con Jan Jonsson, cierra con una discusión sobre el impacto de los efectos primarios y secundarios desde una perspectiva comparada. Los autores ofrecen posibles explicaciones sobre las diferencias encontradas entre países. En principio, se concentran en los factores institucionales, en particular aquellos relacionados con la estratificación y la selectividad de los sistemas educativos. La estratificación hace referencia a la diferenciación jerárquica y temprana entre trayectorias educativas; y la selectividad, a las formas mediante las cuales los estudiantes siguen ciertas trayectorias. En los sistemas educativos estratificados, los estudiantes son asignados a temprana edad en diferentes trayectorias educativas y sus posibilidades de cambiar de trayecto posteriormente son limitadas; mientras que en sistemas poco estratificados las transiciones son más tardías y hay mayor libertad para transitar entre trayectorias distintas. Por otro lado, en aquellos sistemas altamente selectivos, los estudiantes son seleccionados para continuar determinadas trayectorias en función de su desempeño en pruebas de admisión, mientras que los menos selectivos dan flexibilidad a los estudiantes para elegir el trayecto de su preferencia. Dentro del grupo de países estudiados, Alemania y Holanda tienen los sistemas educativos más selectivos y estratificados, mientras que Inglaterra y Estados Unidos cuentan con sistemas menos estratificados y selectivos. En niveles intermedios de estratificación y selectividad se encontrarían Italia, Suecia 
y Dinamarca. Por su parte, el sistema francés es altamente selectivo pero posee un nivel intermedio de estratificación.

De acuerdo con las hipótesis, se esperaría que en países con sistemas más estratificados, los efectos secundarios aumentaran dada la importancia que tiene escoger las trayectorias "adecuadas". En cambio, se esperaría que en los sistemas más selectivos, el papel de los efectos secundarios disminuyera, ya que tendría mayor impacto el rendimiento académico. Sin embargo, debido a que algunos sistemas educativos presentan niveles similares de estratificación y selectividad (tanto alto como bajo), resulta difícil hacer predicciones para cada caso nacional.

Al evaluar las probabilidades de hacer ciertas transiciones en función del origen social, se encontró que los sistemas altamente estratificados y selectivos (Alemania y Holanda) presentan elevados niveles de desigualdad de oportunidades en las primeras transiciones educativas. Por el contrario, países como Estados Unidos e Inglaterra presentan bajos niveles, acorde con lo esperado. Sin embargo, los países que se encuentran en una posición intermedia no presentan un patrón claro. Además, si se atiende a la transición de la educación secundaria alta a la superior, los patrones cambian: los menos desiguales son Inglaterra, Francia y Holanda, mientras Alemania, Suecia y Estados Unidos tienen niveles moderados y semejantes de desigualdad. Como resultado, las características del contexto institucional parecen no ser concluyentes para explicar las diferencias entre países respecto a la magnitud de la desigualdad. Estas diferencias, sostienen los autores, pueden atribuirse a los distintos niveles de desigualdad de condiciones entre países.

Adicionalmente, al analizar la importancia relativa de los efectos secundarios en la desigualdad de oportunidades educativas, se observa que la contribución de los efectos secundarios es alta (de 30\% o más) en todas las transiciones educativas y en todos los países estudiados, excepto para el caso de Inglaterra en la segunda transición, siendo especialmente fuerte en Holanda e Italia (más de $90 \%$ ). Ello indica que aun cuando estudiantes de origen social bajo tengan un buen rendimiento, las elecciones educativas que toman a lo largo de las transiciones limitan considerablemente sus logros educativos futuros.

Al considerar las diferencias en la magnitud de las desigualdades educativas entre países, se encuentra que dichas diferencias están dadas fundamentalmente por el tamaño de los efectos secundarios: cuando estos últimos son altos, la magnitud de la desigualdad de oportunidades educativas es elevada, y viceversa. Por el contrario, la variación del tamaño de los efectos primarios es menor entre países. Este resultado sugiere que las diferencias entre grupos de distinto origen social no residen tanto en las variaciones en el desempeño, sino en el grado en el cual los individuos eligen sus trayectorias. Dicho de otra manera: los estudiantes de origen social desventajado, aun teniendo un alto rendimiento académico, eligen trayectorias educativas de menor alcance; mientras que los individuos de origen social privilegiado, incluso aquellos con bajo desempeño, continúan con trayectorias educativas más demandantes. Como mecanismo explicativo de este resultado, sugieren los autores, estaría no solamente los recursos económicos de las familias, sino también el acceso a información relevante para tomar decisiones sobre los costos y beneficios de las distintas opciones educativas y evaluar las probabilidades de éxito. Así, ante la pregunta que lleva por título esta 
reseña, el libro ofrece una respuesta: son sobre todo las "malas" decisiones las que generan desigualdad de oportunidades educativas.

Por último, cuando se analizan los cambios de la desigualdad educativa en el curso del tiempo, el caso sueco llama especialmente la atención, al ser el único donde se aprecia una disminución significativa en la desigualdad de oportunidades. Esta disminución puede atribuirse, de acuerdo con los autores, a la reducción de los efectos secundarios: una vez controlado el nivel de desempeño, los estudiantes de distintos grupos socioeconómicos tienden a hacer elecciones educativas cada vez más similares.

Los resultados obtenidos tienen además implicaciones de política pública: la importancia de los efectos secundarios encontrada en todos los países y en el curso del tiempo lleva a considerar intervenciones centradas en la eliminación de dichos efectos con el objetivo de reducir la desigualdad de oportunidades. En ese sentido, los autores argumentan que orientar las decisiones a las que se enfrentan los estudiantes y/o sus padres, así como ofrecer becas para aquellos con origen social bajo, constituyen intervenciones con un impacto positivo en la reducción de los efectos secundarios.

En términos generales, el libro ofrece una discusión actualizada y relevante en el área de investigación sobre educación y estratificación social, específicamente en torno al estudio del impacto del origen social en la desigualdad de oportunidades educativas. Su contribución radica en ofrecer evidencia empírica sobre la magnitud de esta desigualdad, sus tendencias de cambio en el curso del tiempo y el impacto de las características institucionales en contextos nacionales. El trabajo no solo hace una cuidadosa revisión de los aspectos teóricos y metodológicos involucrados en la desigualdad de oportunidades, a partir de su descomposición en efectos primarios y secundarios, sino que también identifica los mecanismos explicativos subyacentes así como su aporte al diseño de políticas públicas. Es de destacar además la riqueza y sistematicidad del trabajo comparativo: los conceptos y la metodología son empleados de manera uniforme a lo largo de los capítulos, lo que permite llevar a cabo el ejercicio de comparación pese a las diferencias en la estructura de los sistemas nacionales de educación.

Con todo, el libro se enfoca en un pequeño grupo de países centrales con rasgos relativamente homogéneos, al igual que estudios comparativos previos. La pregunta entonces por la magnitud y los mecanismos de la desigualdad de oportunidades en la periferia sigue en pie: ¿en qué medida la magnitud de los efectos primarios y secundarios es determinada también por el nivel de desigualdad de condiciones, en particular en aquellas naciones donde dicho nivel es alto? Probablemente, en sociedades con una alta desigualdad en la distribución del ingreso, se esperaría un alto grado de desigualdad asociado a un mayor impacto de los efectos primarios y secundarios, dado que los recursos económicos de las familias determinarían de forma más fuerte el desempeño y las opciones educativas que eligen los individuos. La pregunta entonces por la asociación entre el grado de desigualdad de condiciones de un país, las características estructurales de su sistema educativo y la magnitud de las desigualdades educativas continúa siendo una pregunta abierta y urgente para futuras investigaciones. 


\section{Referencias bibliográficas}

Blau, Peter y Otis Duncan. The American Occupational Structure. Nueva York: John Wiley \& Sons, 1967.

Boudon, Raymond. Education, Opportunity, and Social Inequality: Changing Prospects in Western Society. Nueva York: John Wiley \& Sons, 1974.

Bourdieu, Pierre y Jean Passeron. Reproduction in Education, Society and Culture. Londres: Sage Publications, 1977.

Breen, Richard y John Goldthorpe. «Explaining Educational Differentials: Towards a Formal Rational Action Theory". Rationality and Society, Vol. 9, $\mathrm{n}^{\circ}$ 3, 1997: 275-305. doi:10.1177/104346397009003002.

Breen, Richard, Ruud Luijkx, Walter Müller y Reinhard Pollak. «Non-Persistent Inequality in Educational Attainment: Evidence from Eight European Countries». American Journal of Sociology, Vol. 114, $\mathrm{n}^{\circ}$ 5, 2009: 1.475-1.521.

Girard, Alain y Henri Bastide. «La stratification sociale et la démocratisation de l'enseignement». Population, Vol. 18, n 3, 1963: 435-472. doi:10.2307/1526719

Shavit, Yossi y Hans-Peter Blossfeld. Persistent Inequality: Changing Educational Attainment in Thirteen Countries. Boulder: Westview Press, 1993. 